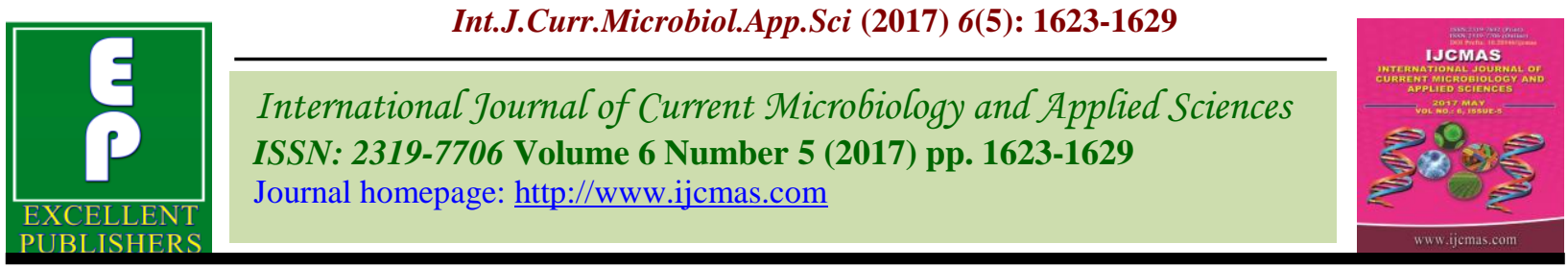

Original Research Article

https://doi.org/10.20546/ijcmas.2017.605.177

\title{
Colonization with Antibiotic-Resistant $E$. coli in Commensal Fecal Flora of Newborns
}

\author{
Amey Tule and Umesh Hassani* \\ Department of Microbiology, NKP Salve Institute of Medical Sciences, \\ Hingna Road, Nagpur, India \\ *Corresponding author
}

\section{A B S T R A C T}

Ke y w o r d s
Multi-drug
resistance,
$\begin{aligned} & \text { Prophylactic } \\ & \text { antibiotics, } \\ & \text { Antibiotic } \\ & \text { determinants. }\end{aligned}$
Article Info
Accepted:
17 April 2017
Available Online:
10 May 2017

Bacterial resistance to antibiotics represents one of the most significant global health challenges of this century. Although the role of commensal bacteria in providing a reservoir of antibiotic resistance has been acknowledged for some time these bacteria have not been studied extensively. The purpose of the present paper is to prospectively determine the presence of Multi drug resistant $E$. coli in commensal fecal flora of Newborns who are not receiving any antibiotics. After receiving parental consent 30 neonates less than 1month of age of either sex, hospitalized in the pediatric unit of NKP Salve Institute of Medical Sciences, not recently exposed to antibiotics were enrolled along with 30 neonates less than 1 month of age admitted in NICU on treatment with various antibiotics. E. coli isolates from Stool samples were identified by standard biochemical tests \& Antibiotic susceptibility was tested by the Kirby-Bauer disk diffusion method on Mueller-Hinton agar. The Antibiogram of both the groups was compared to determine whether antibiotic therapy results in increase in the percentage of drug resistant $E$. coli population in the commensal fecal flora. In the newborns, who were not given antibiotics, resistance to Ampicillin was $100 \%$, followed by Co-trimoxazole 27(96.42\%). Highest sensitivity was observed to Amikacin 24(85.71\%) followed by Gentamicin 16(57.14\%).60\% isolates were resistant to more than 3 drugs. In Newborns who were on various antibiotics, $100 \%$ resistance was seen to Ampicillin and Ceftriaxone followed by Co-trimoxazole 27(96.42\%). Highest sensitivity was observed with Chloramphenicol 13(46.42). 11 isolates (39.28\%) were sensitive to Amikacin and Tetracycline each. 90\% isolates were resistant to more than 3 drugs.

\section{Introduction}

Bacterial resistance to antibiotics represents one of the most significant global health challenges of this century. Infections with multiple antibiotic-resistant bacteria have been increasing at an alarming rate owing to the widespread dissemination of antibioticresistance determinants (Collignon, 2002; O'Brien, 2002; Alekshun et al., 2006; Marshall et al., 2009) Although the role of commensal bacteria in providing a reservoir of antibiotic resistance has been acknowledged for some time (Alekshun et al., 2006; Marshall et al., 2009; Hawkey, 1986), these bacteria have not been studied extensively. Escherichia coli constitutes only a minor fraction of the bacterial flora found in the human gastrointestinal tract but is none the less an important reservoir. Increasing, incidence of high antibiotic resistance in commensal E. coli from healthy children and 
adults from many countries has been documented by many recent studies (Jannine et al., 2010).

Hospital-associated Gram-negative bacterial colonization is acquired by newborns admitted to the intensive care unit throughout their NICU stay. It is most probably transmitted from the environmental flora via the hands of caregivers. Colonization specifically by antibiotic-resistant bacilli follows a similar pattern, suggesting that such bacteria make up part of the modern NICU ecology and are acquired by infants similar to susceptible microorganisms. Although some clinically-undetected cross-transmission of resistant bacilli occurs during non-outbreak periods, most colonizing antibiotic-resistant bacilli are unique to each infant (Toltzis, 2003).

Outbreaks with multidrug resistant bacteria usually result in treatment failure and also associated with increased mortality and higher costs. Adequate infection control practice, to limit horizontal spread of the bacteria and an early empirical antibiotic treatment effective against the causative bacteria is largely decisive for the outcome of neonatal septicemia (Toltzis, 2003).

The purpose of the present paper is to prospectively determine the presence of Multi drug resistant $E$. coli in commensal fecal flora of neonates who are exposed to antibiotics versus those who are not exposed to antibiotics and also to determine the extent of problem in our region.

\section{Materials and Methods}

After clearance from Institutional ethics Committee, a cross sectional study was done. All neonates admitted in the NICU and SICU of NKP Salve Institute of Medical Sciences and Lata Mangeshkar Hospital, Digdoh from June 2014 to September 2014 (3 months) was enrolled in the study (As the study was part of ICMR-STS project)

Total 60 neonates were enrolled in the study, after receiving parental consent

\section{Inclusion criteria}

All neonates less than 1 month of age admitted in the hospital.

\section{Exclusion criteria}

Baby born to immune compromised mother.

The neonates were divided into 2 groups

Group A: 30 neonates less than 1month of age of either sex, hospitalized in the pediatric unit of NKP Salve Institute of Medical Sciences, not recently exposed to antibiotics were enrolled along

Group B: 30 neonates less than 1month of age admitted in NICU on treatment with various antibiotics.

\section{Methods}

Medical history (prior hospitalization, prior invasive devices used, Mode of delivery), antibiotic therapies, diagnosis on admission and discharge was recorded in case record form. Patient Stool samples were collected and immediately transported to microbiology laboratory.

\section{Laboratory methods}

Stool samples were immediately plated onto Mac-conkeys agar. The plates were kept in incubation at $37^{\circ} \mathrm{C}$ for 24 - to 48 hours. The $E$. coli isolates were identified by Gram staining and by standard biochemical tests. E. coli isolate from each stool sample was subjected to antimicrobial susceptibility testing using Kirby-Bauer disc diffusion method. CLSI interpretative criteria for susceptibility and 
resistance were used, ATCC E. coli 25922 was simultaneously tested as control with each batch of antimicrobial susceptibility testing performed (Clinical Laboratory Standards Institute, 2011). After verifying the results of the standard strain, test sample results were interpreted. Zone diameters were read using antibiotic zone scale (Himedia, Mumbai, India).

Following antibiotics discs were tested (HiMedia, Mumbai), Ampicillin (10mcg), Cotrimoxazole $(25 \mathrm{mcg})$, Gentamicin $(30 \mathrm{mcg}$ ), Amikacin (30 mcg), Tetracycline $(30 \mathrm{mcg})$, Chloramphenicol (30 mcg), Ceftriaxone (30 mcg), Ciprofloxacin (5 mcg).

\section{Data analysis}

The above collected data was analyzed by proportion rate method.

\section{Results and Discussion}

The number of $E$. coli isolates obtained from the neonates who were not exposed to antibiotics was 28 (Group A) and similar number of $E$. coli isolates were obtained from the neonates who were admitted to NICU and were on various antibiotics (Group B). Two stool samples did not show growth of $E$. coli from both the groups.

In neonates belonging to group A, all isolated E. coli were resistant to Ampicillin (100\%), followed by co-trimoxazole 27(96.42\%). Most of the isolates were sensitive to $d$ to Amikacin 24(85.71\%) followed by Gentamicin 16(57.14\%). Multi drug resistance (more than 3 drugs) was seen in sixty percent of isolates (Table 1).

In neonates belonging to group $\mathrm{B}$, all isolates were resistant to E. coli and Ceftriaxone, followed by co-trimoxazole 27 (96.42\%).
Highest sensitivity was observed with Chloramphenicol 13 (46.42\%).

Eleven isolates (39.28\%) were sensitive to Amikacin and Tetracycline each. Multi drug resistance (more than 3 drugs) was seen in Ninety percent isolates (Table 2).

Almost all the neonates admitted to a NICU belonging to group $\mathrm{B}$ were on various antibiotics (Table 3) shows the antibiotics administered to the neonates during their admission. The initial empirical antibiotics administered were Vancomycin and Ceftriaxone. The antibiotics were changed after culture and sensitivity reports.

Fifty-seven neonates in the present study were delivered by c-section and three neonates were by normal delivery. All the mothers who went through $\mathrm{C}$-section were given Injection Ceftriaxone and Injection Metronidazole as prophylactic antibiotics one hour before operation (Table 4).

Irrational use of antibiotics as prophylaxis or for empirical use results in exposure of microorganism to antimicrobial agents. Such use of an antimicrobial agent selects for overgrowth of a bacterial strain that has a gene expressing resistance to the agent. The levels of resistance to antimicrobial agents at any time and place may therefore reflect in part the total number of bacteria in the world exposed to antimicrobials up until then. There is widespread indirect dissemination of antimicrobial resistance from varied sources, this is evident from studies on microbial population biology, microbial genetics, and clinical and epidemiological observations (Karine et al., 2004).

In the present study $100 \%$ resistance was observed to Ampicillin and 96\% resistance was observed to Co-trimoxazole in Group I. 
Most of the isolates were sensitive to Amikacin (Table 1).

Dyar et al., conducted a study in rural community of Vietnam, high degree of resistance was found in the $E$. coli isolated from feces to Ampicillin (65\%), Tetracycline (74\%) and Co-trimoxazole (68\%). Resistance to Chloramphenicol and Nalidixic acid was significantly low (10) In the study by Shanahan et al., $88.6 \%$ E. coli were resistant to Ampicillin, followed by Trimethoprim (74.2\%). Chloramphenicol resistance was found to be lower at $52.6 \%$ while the carriage of Gentamicin and Nalidixic acid resistance were lower still at $7.5^{\circ} \%$ and $10.2 \%$ respectively.(11) In the rural community of French Guyana, Karine grenet et al., (2004) observed that E. coli isolates were mostly resistant to Amikacin (55\%) and Tetracycline (63\%) followed by Streptomycin (45\%). Twenty nine and twenty four percent isolates were resistant to Co-trimoxzole and Cholramphenicol respectively. All isolates were sensitive to third generation Ceftriaxone and Nalidixic acid.

In a study by Garcia et al., (2011), total 220 strains were recovered from the fecal specimens $(61.8 \%$ from acute diarrhoea and $38.2 \%$ from without diarrhoea). The isolates showed high resistance rates against ampicillin, tetracycline, and sulfamethoxazole-trimethoprim.

In another study, by Oluyege et al., (2015) The E. coli isolates exhibited $90.2 \%$ and $88.2 \%$ resistance to amoxicillin and tetracycline respectively while over $75 \%$ were resistant to cotrimoxazole and augmentin. Ninety percent $(90 \%)$ of $E$. coli isolated had multiple resistance to three or more antibiotics. There was carriage of multiple resistant commensal E. coli irrespective of exclusive or inclusive mode of feeding, or previous history of use of antibiotics or other parenteral drugs. Carriage of multiply resistant commensal E. coli was present even in infants with no history of use of any drug.

In a meta analysis by Bryce et al., (2016), 34 research paper of carriage of antibiotic resistant fecal E. coli were analysed along with comparison of economic status on basis of OECD status. The study showed high rates of faecal $E$. coli resistance in asymptomatic children, the resistance rate was higher in non-OECD countries and association was found between primary care prescribed antibiotics and resistance lasting for up to 3 months post-prescribing. Sheidman et al., (2009) demonstrated that $32 \%$ of E. coli was resistant to more than three antibiotics.

In a study by Shakya et al., (2013) for antibiotic resistance patterns in $E$. coli isolates from stool samples of children aged 3-14 years from Ujjain, antibiotic resistance patterns in E. coli isolates, overall, $72 \%$ of isolates were resistant to at least one antibiotic and $33 \%$ were multi-drug resistant. Proportions of isolates resistant to various antibiotics were, nalidixic acid, (45\%), tetracycline (37\%), ampicillin (37\%), sulfamethoxazole/trimethoprim (29\%) and amoxicillin/clavulanic acid (29\%). High rates of cross-resistance were seen for $15(83 \%)$ of the antibiotics studied. No isolates were resistant to imipenem.

Thus Studies done by various authors have demonstrated higher resistance to Ampicillin and Co-trimoxazole in the commensal fecal flora. The sensitivity towards Aminoglycosides and Fluroquinolone was varying from $80 \%$ to $50 \%$.

The findings in our study for Group 1 are similar to most of the other studies which have studied $E$. coli isolates in fecal flora from community and shows that most of the E. coli isolates in community show high degree of resistance to Ampicillin and Co- 
trimoxazole. The studies also show that multidrug resistance (resistance to more than 3 drugs) is common phenomenon in various parts of the world.

In the present study in Group II, most of the isolates were resistant to Ampicillin and Ceftriaxone. The resistance to Amikacin and Ciprofloxacin was also significantly increased in this group as compared to Group I (Table 2) $(\mathrm{p}<0.5)$. Study conducted in Indonesia at Fatimawati hospital by Maksum Radji, Siti Fauziah, Nurgani Aribinuko for Antibiotic sensitivity pattern of bacterial pathogens in the intensive care unit showed resistance pattern as Ceftriaxone (46\%), Amikacin (15.4\%), Gentamycin (38\%), Ciprofloxacin (46.2\%). In a study performed by Todisoa Andriatahina et al., (2010) fecal carriage of Multi drug resistant E. coli was detected in $21.2 \%$ of 244 infants on admission and $57.1 \%$ of 154 on discharge, after more than 48 hours of hospitalization ( $\mathrm{p}<0.001)$. E. coli which was ESBL-positive showed high degree of resistance to Trimethoprim-Sulfamethoxazole (91.3\%), Gentamicin (76.1\%), Ciprofloxacin (50.0\%), but not to Amikacin and Imipenem. The increased prevalence of carriage during hospitalization was related to standard antimicrobial therapy.

Table.1 Antibiogram of E. coli isolates obtained from the neonates who were not exposed to antibiotics

\begin{tabular}{|l|c|}
\hline \multicolumn{1}{|c|}{ ANTIBIOTICS } & RESISTANT N=28 \\
\hline AMPICILIN & $28(100 \%)$ \\
\hline CO-TRIMOXAZOLE & $27(96.42 \%)$ \\
\hline CEFTRIAXONE & $12(42.85 \%)$ \\
\hline GENTAMICIN & $12(42.85 \%)$ \\
\hline AMIKACIN & $4(14.28 \%)$ \\
\hline CIPROFLOXACIN & $16(57.14 \%)$ \\
\hline TETRACYCLINE & $21(75 \%)$ \\
\hline CHLORAMPHENICOL & $16(57.14 \%)$ \\
\hline \multicolumn{2}{|c|}{ TOTAL } \\
\end{tabular}

Table.2 Antibiogram of E. coli isolates obtained from the neonates admitted to NICU and were on various antibiotics

\begin{tabular}{|c|c|}
\hline ANTIBIOTICS & RESISTANT $(\mathrm{N}=28)$ \\
\hline AMPICILIN & $28(100 \%)$ \\
\hline CO-TRIMOXAZOLE & $22(78.57 \%)$ \\
\hline CEFTRIAXONE & $28(100 \%)$ \\
\hline GENATAMICIN & $20(71.42 \%)$ \\
\hline AMIKACIN & $17(60.71 \%)$ \\
\hline CIPROFLOXACIN & $21(75.00 \%)$ \\
\hline TETRACYCLINE & $17(60.71 \%)$ \\
\hline CHLORAMPHENICOL & $15(53.57 \%)$ \\
\hline TOTAL & $28(100 \%)$ \\
\hline
\end{tabular}


Table.3 Antibiotics administered to neonates admitted to NICU

\begin{tabular}{|c|c|}
\hline Name of Antibiotics & Percent of newborns \\
\hline Vancomycin & $100 \%$ \\
\hline Ceftriaxone & $50 \%$ \\
\hline Amikacin & $70 \%$ \\
\hline Meropenem & $100 \%$ \\
\hline
\end{tabular}

Table.4 Antibiotic administered to mother during cesarean section as prophylactic antibiotics

\begin{tabular}{|c|c|}
\hline Name of antibiotics & No (\%) \\
\hline Ceftriaxone & $100 \%$ \\
\hline Metronidazole & $100 \%$ \\
\hline
\end{tabular}

Dethlefsen et al., (2008) in a study demonstrated that administration of ciprofloxacin, a commonly used antibiotic with little activity against obligate anaerobic bacteria, altered the abundance of roughly one third of intestinal bacteria in three healthy volunteers, with a significant loss of microbial diversity during treatment.

Most of the studies done in the hospitalized population have shown that the E. coli in the commensal flora of the hospitalized patients acquires resistance due to selective pressure of the antibiotics. The antibiogram depends on the antibiotics routinely used in the hospital.

In our hospital the antibiotics used for prophylaxis were Cephalosporin (Ceftriaxone) and Glycopeptides (Vanco-mycin). We have tested the isolates for their resistance against cephalosporin and our data shows that the number of isolates resistant to Ceftriaxone is significantly more in the Group II than in the Group I ( $p<0.5)$

Also the use of Ceftriaxone as prophylactic antibiotics in the mothers may have influenced the resistance pattern to the E. coli in commensal fecal flora (Table 4)

The isolates in group 2 were ninety percent multidrug resistant (Table 2). Fecal E. coli is regarded as a useful indicator of the spread of acquired antibiotic resistance genes in the community, Commensal Escherichia coli can act as reservoirs of resistance genes in the human gut. These resistant genes might be rapidly transferred to other commensal or pathogenic organisms. Hence proper antibiotic policy and restricted use of higher antibiotics is need of hour.

In conclusion, our study demonstrated the high prevalence of resistance to individual antibiotics in commensal $E$. coli isolated from both groups of newborns admitted in neonatal intensive care unit. The increased empirical use of antibiotics has caused multidrug resistant $E$. coli to emerge even in the commensal flora of community hence it is imperative to use antibiotics judiciously. As the study period was limited, number of isolates studied was less hence study with larger population is required to completely assess the impact of antibiotics on commensal fecal flora.

\section{References}

Alekshun, M.N. and Levy, S.B. 2006. Commensals upon us, Biochem. Pharmacol., 71(7): 893-900.

Bryce, A., Costelloe, C., Hawcroft, C., Wootton, M., Hay, A.D. 2016. Faecal carriage of antibiotic resistant Escherichia coli in asymptomatic children and associations with primary care antibiotic prescribing: a systematic review and meta-analysis. $B M C$ Infect. Dis., 16(359): 1697-6.

Clinical Laboratory Standards Institute. 2011. 
Performance Standards for Antimicrobial Susceptibility Testing: Twentieth informational supplement. CLSI document M100-S21.PA, USA: Wayne.

Collignon, P.J. 2002. Antibiotic resistance. Med. J. Aust., 177(6): 325-329.

Dethlefsen, L., Huse, S., Sogin, M.L., Relman, D.A. 2008. The pervasive effects of an antibiotic on the human gut microbiota, as revealed by deep $16 \mathrm{~S}$ rRNA sequencing. PLoS Biol., 6: e280.

Hawkey, P.M. 1986. Resistant bacteria in the normal human flora. J. Antimicrob. Chemother., 18(Supplement C): 133-139.

Jannine, K., Bailey, Jeremy, L., Pinyon, et al. 2010. Commensal Escherichia coli of healthy humans: a reservoir for antibioticresistance determinants. J. Med. Microbiol., 59: 1331-1339.

Karine Grenet, Didier Guillemot, Vincent Jarlier, et al. 2004. Antibacterial Resistance, Wayampis Amerindians, French Guyana. Emerg. Infect. Dis., 10(6): 1150-1153.

Maksum Radji, Siti Fauziah, Nurgani Aribinuko. 2011. Antibiotic sensitivity pattern of bacterial pathogens in the intensive care unit of Fatmawati Hospital, Indonesia, Asian Pac. J. Trop. Biomed., 1(1): 39-42.

Marshall, B.M., Ochieng, D.J. and Levy, S.B. 2009. Commensals: underappreciated reservoir of antibiotic resistance. Microbe, Vol 4(5): 231- 238.

O'Brien, T.F. 2002. Emergence, spread, and environmental effect of antimicrobial resistance: how use of an antimicrobial anywhere can increase resistance to any antimicrobial anywhere else. Clin. Infect. Dis., 34 (Supplement 3): S78-S84.

Oliver James Dyar, Nguyen Quynh Hoa, et al. 2012. High prevalence of antibiotic resistance in commensal Escherichia coli among children in rural Vietnam. BMC Infect. Dis., 12: 92.

Oluyege, A.O., Ojo-Bola, O. and Oludada, O.E. 2015. Carriage of Antibiotic Resistant Commensal E. coli in Infants below 5 Months in Ado-Ekiti. Int. J. Curr. Microbiol. App. Sci., 4(5): 1096-1.

Patrícia, G., Garcia, Vânia L. Silva, and Cláudio, G., Diniz. 2011. Occurrence and Antimicrobial Drug Susceptibility Patterns of Commensal and Diarrheagenic Escherichia coli in Fecal Microbiota from Children with and Without Acute Diarrhea. The J. Microbiol., Vol 49(I) p 46-52.

Pragya Shakya, Peter Barrett, Vishal Diwan et al. 2013. Antibiotic resistance among Escherichia coli isolates from stool samples of children aged 3 to 14 years from Ujjain, India. BMC Infect. Dis., 13: 477.

Seidman, J.C., K.P. Anitha, R. Kanungo, A.L. Bourgeois, and C.L. Coles. 2009. Risk factors for antibiotic-resistant $E$. coli in children in a rural area. Epidemiol. Infect., 137(6): 879-888.

Shanahan, P.M.A., B.A. Wylie, et al. 1993. The prevalence of antimicrobial resistance in human faecal flora in South Africa. Epidemiol. Infect., 111(2): 221-228.

Todisoa Andriatahina, Frederique Randrianirina, Eliosa Ratsima Hariniana, et al. 2010. High prevalence of fecal carriage of extendedspectrum $\quad \beta$-lactamase-producing Escherichia coli and Klebsiella pneumoniae in a pediatric unit in Madagascar $B M C$ Infect. Dis., 10: 204.

Toltzis, P. 2003. Colonization with antibioticresistant Gram-negative bacilli in the neonatal intensive care unit. Minerva Pediatr., 55(5): 385-93.

\section{How to cite this article:}

Amey Tule, Umesh Hassani. 2017. Colonization with Antibiotic-Resistant E. coli in Commensal Fecal Flora of Newborns. Int.J.Curr.Microbiol.App.Sci. 6(5): 1623-1629.

doi: https://doi.org/10.20546/ijcmas.2017.605.177 